\title{
Role of Husband's Age in Percentage of Eggs that get Fertilized in Invitro Fertilization Cycle
}

\author{
Article by Monisayo Olayemi Komolafe ${ }^{1}$, Komolafe Johnson Olusanmi ${ }^{2}$ \\ ${ }^{1}$ Lautech Teaching Hospital, Osogbo \\ ${ }^{2}$ Department of Obstetrics \& Gynaecology \\ Lautech Teaching Hospital, Osogbo \\ E-mail: monisayokomolafe@gmail.com ${ }^{1}$,komosayo@gmail.com²
}

\begin{abstract}
Background: Female age is a known independent index in determining outcome of fertility treatment with ovulatory and fertility potentials declining rapidly after age 35 however it an assumed fact that men are capable of spermatogenic activity beyond age 60 with world oldest father procreating at age 97. (Myers, 1972)

Objectives: Determine if age affected semen concentration and motility of male partners undergoing invitro fertilization.

Determine if age of male partners had influence on percentage of oocytes that are fertilized following invitro fertilization cycles.

Method: A descriptive retrospective review of data of IVF cycles performed between July 2015 and June 2017. Inclusion criteria was that age of oocytes producer must be less than 36, post sperm wash count of at least 10million per milliliters and 15\% active motile sperm. Exclusion criteria were female age of oocyte producer more than 35, incomplete relevant data.

Results: One hundred and twenty cycles met inclusion criteria. Mean husband age was 45.70 ( \pm 7.07$)$. Mean percentage oocyte fertilized among the group was $51.95 \%( \pm 29.85 \%)$. Mean percentage of oocytes fertilized among husband equal to or less than 50 was $54.21 \%$ ( $\pm 28.87 \%)$ while mean percentage of oocytes fertilized among husband age 51 and above was $45.20 \%$ ( \pm 32.20$)$, the observed difference being statistically significant $\left(p=0.0000 ; X^{2}=90.133\right)$.

Conclusion: There is a significant drop in ability of men above 50years to fertilize oocytes compared to men below 51 years of age during invitro fertilization cycles.
\end{abstract}

Keywords: In-vitro Fertilization, Intra uterine insemination.

\section{Abbreviation}

BNSc Bachelors Degree Nursing Science

IUI Intra uterine insemination

IVF In-vitro fertilization

O\&G Obstetrics and Gynecolog

\section{Introduction}

Infertility is a major problem in our culture and society at large. Infertile couple are seen as abnormal, unfortunate and most of the time the woman is seen as the cause of delay in child bearing while the man is always seen as free of all abnormality hence does not even present themselves for evaluation in the fertility centers of our hospital units.

It has also been observed in our society that academic and economic pursuit's leads to late marriage which has been documented to affect the females. In couples younger than age 30 who are generally healthy, $20 \%$ to $37 \%$ are able to conceive in the first 3 months (American Society for Reproductive 
DOI: $10.21522 /$ TIJNR.2015.03.02.Art013

ISSN: $2520-3126$

Medicine, 2012), but males are believed to be fertile throughout their life time. Therefore, this study wants to investigate if there is any significance in fertilization rate in relation to paternal age.

It has been established that one in every six couple is infertile.

Infertility is defined clinically in women and men who cannot achieve pregnancy after 1 year of having intercourse without using birth control, and in women who have two or more failed pregnancies. Studies suggest that after 1 year of having unprotected sex, $15 \%$ of couples are unable to conceive, and after 2 years, $10 \%$ of couples still have not had a successful pregnancy. (Practice Committee of the American Society for Reproductive Medicine in collaboration with the Society for Reproductive Endocrinology and Infertility, 2008), (Male Infertility Best Practice Policy Committee of the American Urological Association and Practice Committee of the American Society of Reproductive Medicine, 2006).

For conception to take place a man's sperm must combine with the woman's egg. Sperm are made and stored in the testicles, which are ejaculated by the penis to deliver sperm to the female reproductive tract during sexual intercourse.

External genital organs: Penis-The penis is the male intromittent organ. It has a long shaft and an enlarged bulbous-shaped tip called the glans penis, (https://prezi.com/i0z5miveenwg/human-malereproductive-system/ Oct 1, 2013) which supports and is protected by the foreskin. When the male becomes sexually aroused, the penis becomes erect and ready for sexual activity. Erection occurs because sinuses within the erectile tissue of the penis become filled with blood. The arteries of the penis are dilated while the veins are compressed so that blood flows into the erectile cartilage under pressure. (https://en.wikipedia.org/wiki/Male_reproductive_system) The penis is supplied by the pudendal artery.

Scrotum-The scrotum is a pouch-like structure that hangs behind the penis. It holds and protects the testicles. It also contains numerous nerves and blood vessels. During times of lower temperatures, the Cremaster muscle (https://hyattractions.wordpress.com/2012/11/.. ./male-and-female-reproductivesystem, 2012) contracts and pulls the scrotum closer to the body, while the Dartos muscle gives it a wrinkled appearance; when the temperature increases, the Cremaster and Dartos muscles relax to bring down the scrotum away from the body and remove the wrinkles respectively.

The scrotum remains connected with the abdomen or pelvic cavity by the inguinal canal. (https://hyattractions.wordpress.com/2012/11/.../male-and-female-reproductive-system ， 2012) (The spermatic cord, formed from spermatic artery, vein and nerve bound together with connective tissue passes into the testis through inguinal canal.)

Epididymis-The epididymis, a whitish mass of tightly coiled tubes cupped against the testicles, (https://en.wikipedia.org/wiki/Male_reproductive_system) acts as a maturation and storage for sperm before they pass into the vas deferens, that carry sperm to the ampullary gland and prostatic ducts.

Vas deferens-The vas deferens, also known as the sperm duct, is a thin tube approximately 30 centimetres $(0.98 \mathrm{ft})$ long that starts from the epididymis to the pelvic cavity. It carries the spermatozoa from the epididymis to ejaculatory duct.

To conceive a child, a man's sperm must combine with a woman's egg. The testicles make and store sperm, which are ejaculated by the penis to deliver sperm to the female reproductive tract during sexual intercourse. The most common issues that lead to infertility in men are problems that affect how the testicles work. Other problems are hormone imbalances or blockages in the male reproductive organs. In about $50 \%$ of cases, the cause of male infertility cannot be determined. (Jose-Miller, 2007)

A complete lack of sperm occurs in about $10 \%$ to $15 \%$ of men who are infertile. (American Urological Association, 2008). A hormone imbalance or blockage of sperm movement can cause a lack of sperm. In some cases of infertility, (samadiroboticsfoundation.org/what-is-male-infertility/ Nov 18, 2016) a man produces less sperm than normal. The most common cause of this condition is varicocele, an enlarged vein in the testicle. (Damilola F, 2015) Varicocele is present in about $40 \%$ of men with infertility problems. (American Urological Association, 2008)

Sperm specimen should be collected by masturbation and the ejaculate produced into a sterile glass that has been checked for sperm toxicity. As soon as the seminal plasma has liquefied, the specimen 
should be analyzed according to the WHO guidelines and prepared for sperm isolation. Sperm can be prepared by Simple Washing and Dilution. Repeat centrifugation, usually two or three times in total, is often used to ensure removal of contaminating seminal plasma. The centrifugation is usually performed at 200-300 g and it should certainly be performed at centrifugal forces less than $800 \mathrm{~g}$ (Jeulin C, 1982).

\section{Research design}

A descriptive retrospective review of data of IVF cycles performed between July 2015 and June 2017. Inclusion criteria was that age of oocytes producer must be less than 36, post sperm wash count of at least 10million per milliliters and $15 \%$ active motile sperm. Exclusion criteria were female age of oocyte producer more than 35 , incomplete relevant data.

\section{Research population}

A total of three hundred and five cycles were recorded. All the respective folders were retrieved in batches and parameters retrieved with predetermined data sheet. Inclusion criteria included age of the woman who had oocyte retrieval must be less than 36years, the semen parameters after sperm washing preparatory for invitro fertilization must be at least ten million per milliliter with at least $15 \%$ of them actively and progressively motile. All cases with incomplete data for example age of husband not indicated or percentage motility of the sperm after sperm preparation omitted were all excluded from analysis.

\section{Research setting}

A private fertility specialist hospital 'Ayomide Womens' health IVF centre and fertility hospital. The hospital is located in Osogbo the state capital of Osun state. This hospital major mainly in various aspects of fertility care and investigations.

The hospital has three main theatres first occupying the ground floor of the hospital is the laparoscopy and hysteroscopy theatre where closed surgeries and diagnostic laparoscopy is carried out.

The second theatre is the IVF theatre where follicular aspiration is done for IVF treatments. Attached to the IVF theatre is the andrology laboratory where the embryologist work on the aspirated eggs and semen preparation is carried. The third theatre is where various obstetrics and gynecological open surgeries such as caesarian section, myomectomy, subtotal abdominal hysterectomy ,Total abdominal hysterectomy, vaginal hysterectomy, adhesiolysis for intrauterine and pelvic adhesion.

Other investigations and procedures includes Hormonal assay, follicular tracking, abdominal and transvaginal scans, intra uterine insemination-IUI. Ante natal care is rendered to pregnant women on Mondays while Gynecological clinics holds on Wednesdays and Fridays of every week.

The hospital is covered by a consultant Obstetrician and gynecologist who is also a fertility specialist.

Other doctors are an O\&G consultant, a senior medical officer and two medical officers. The hospital has six Registered Nurses and midwives, three with BNSc.

The hospital has five private rooms, four bedded post natal ward, four bedded post embryo transfer ward, a labour ward, one neonatal ward with two phototherapy kits. All resuscitative measures such as multi parameter monitor, 100percent oxygen, suctioning machine etc., are always available to meet clients need. The hospital has recorded several successful IVF pregnancies with deliveries of live infants

The hospital has two certified embryologist and two trainees. There are four ward orderlies and two security men

\section{Research sampling and sampling technique}

Convenience sampling of all IVF cases done that met inclusion criteria of the 305 listed cases were one hundred and twenty. 
DOI: $10.21522 /$ TIJNR.2015.03.02.Art013

ISSN: $2520-3126$

\section{Method of data collection}

The list and embryology data sheets of invitro fertilization cycles conducted from $1^{\text {st }}$ of July 2015 to $30^{\text {th }}$ day of June 2017 was retrieved from the director of embryology laboratory. A total of three hundred and five cycles were recorded. All the respective folders were retrieved in batches and parameters retrieved with predetermined data sheet. Inclusion criteria included age of the woman who had oocyte retrieval must be less than 36years, the semen parameters after sperm washing preparatory for invitro fertilization must be at least ten million per milliliter with at least $15 \%$ of them actively and progressively motile. All cases with incomplete data for example age of husband not indicated or percentage motility of the sperm after sperm preparation omitted were all excluded from analysis. Total number that met inclusion criteria of the 305 listed cases were one hundred and twenty. Semen analysis is done in the facility using world health organization criteria (WHO)

\section{Method of data analysis}

The retrieved data were entered into SPSS version 17.0. The data was sorted by age in ascending order, age of husband variable was recoded into different variable of age group less than 40years, age 41-45, 4650, 51-55 and above 55 years. Descriptive analysis was subsequently carried out yielding frequencies, mean, and standard deviations of numerical data. Cross tabulations of percentage oocyte fertilized against that of age groupings were then done with determination of level significance of observed difference using chi square.

\section{Ethical consideration}

Permission to use stored data for the purpose of this research work was gotten by a formal request to the ethical review unit of the hospital which gave her consent.

\section{Result}

A total of one hundred and twenty cycles met the inclusion criteria set for this study out of the three hundred and five cycles conducted during the two year period. The mean age of husband in this study is 45.70 ( \pm 7.07$)$. Almost fifty percentage of the male partners were in the fourth decade (Table 1a) with three quarters of them aged below 51years (Table 1b). The age range was from minimum of 33 to maximum of 64years. Mean semen count post sperm washing preparatory for invitro fertilization among the studied male partners was one hundred and twenty million per milliliter $\left(120 \times 10^{6} / \mathrm{ml} \pm 97.91\right)$ while mean sperm motility among reviewed cases was $44.67 \% \pm 16.87$ and mean oocyte production among the women reviewed was $9.9 \pm 3.87$.

Mean percentage oocyte fertilization in this review was $51.95 \%( \pm 29.85)$ with zero fertilization in fifteen $(12.5 \%)$ of cases reviewed while hundred percent fertilization was seen in only three of the cases $(2.5 \%)$. When the age groupings were cross tabulated against percentage oocyte fertilized, increasing age was found to be inversely proportionally related percentage egg fertilized especially after age of 50 years. The observed difference among the age groupings were found to be statistically significant (Table $2 \& 3$ ).

\section{Discussion}

Semen Parameters and age: Mean semen concentration among the studied male partners was 120 million per milliliter. The value appears high compared to average expected value for post semen wash count to perform intrauterine insemination (IUI) or invitro fertilization which is put at 10 million per milliliter(Ok E.K, 2013). This is not unexpected as men with semen adjuged not to meet minimum WHO semen parameters of semen concentration of 15 million per milliliter (Morshedi M, 2010) were excluded to remove confounding factors that may affect ability to fertilize oocytes apart from husband age.

Semen concentration showed a direct proportional increase with increasing age and this became distinctly noticeable in men over 55 years, this is in keeping with findings of Brahem et al (Brahem S, 2011) and (Andolz, 1999) compared to others who recorded no change or minimal decrease in sperm 
concentration) (Auger J,1995) (Schwartz D,1983). Andolz et al in a study of study of 20,411 men found a statistically significant increase in concentration of $0.7 \%$ per year of age amounting to an increase in concentration of $14 \%$ over a 20 -year period.

Mean semen motility among reviewed men in this study was $44.67 \%$. This also as expected by the design of this study is expected to be above the minimum motility of $15 \%$ progressively motile sperm for IUI or IVF (Michelmann H.W, 1995) therapy. Sperm motility in relation to advancing age in this review showed inverse relationship. This agrees with general consistent findings of reduction in motility with advancing age in many studies (Wu Y, 2015), (Harris I.D, 2011), (Auger J, 1995).

\section{Oocytes fertilization and age}

Mean oocyte fertilization in this review was $51.95 \%$ with zero fertilization in fifteen $(12.5 \%)$ of cases reviewed. Mean oocyte fertilization of $54.21 \%$ found among male partners who are 50years or less dropped to $45.21 \%$ among those who are 51years or over. Yixuan et al concluded that fertilization rates of oocytes is not altered by paternal age. (Sloter E, 2006). There is paucity of papers looking at effects of paternal age on fertilization as most papers invariably puts advanced maternal age as main cause of failure of fertilization. This study with is suggesting that there may be a role for paternal age in determination of oocytes fertilization.

\section{Recommendation}

More studies to explore role of paternal age in occyte fertilization to be conducted. Men like women folks need to attend to reproductive challenges early.

\section{Conclusion}

There is a significant drop in ability of men above 50years to fertilize oocytes compared to men below 51years of age during invitro fertilization cycles.

\section{Figures and tables}

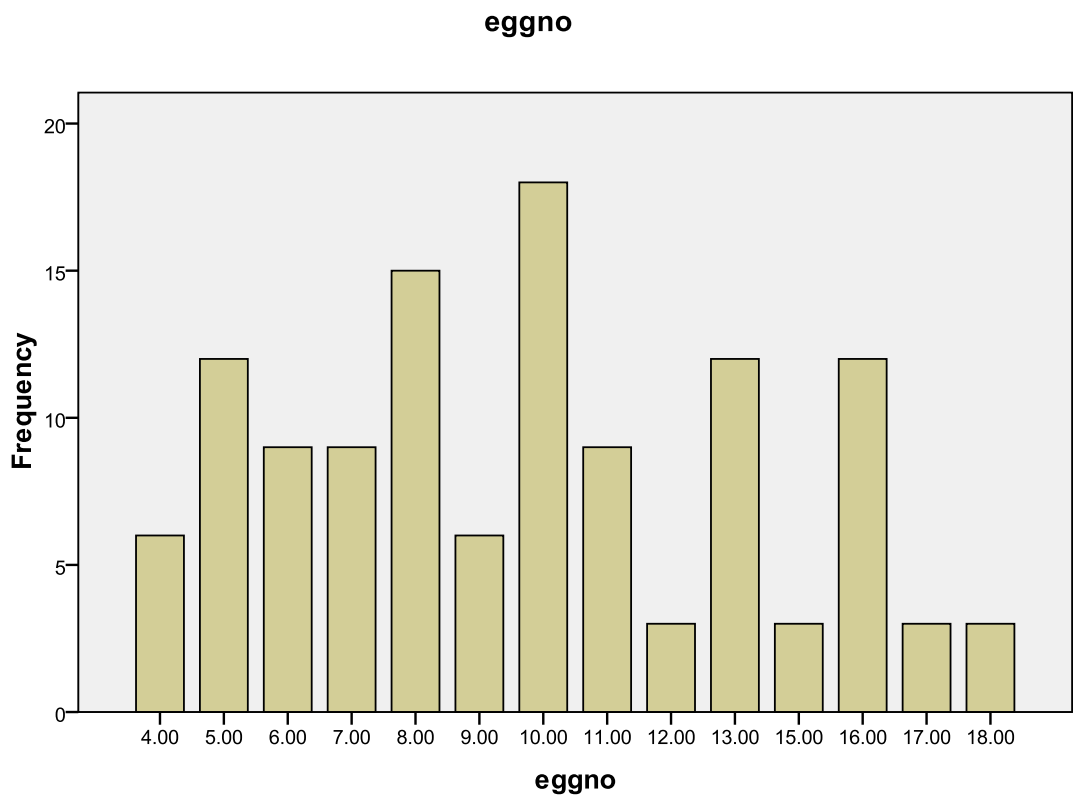

Figure 1. Distribution of oocytes retrieved from female population of the study 
DOI: $10.21522 /$ TIJNR.2015.03.02.Art013

ISSN: $2520-3126$

Table 1. Age distribution of male partners of studied IVF cycles

$1 \mathrm{~A}$.

\begin{tabular}{|l|l|l|}
\hline S/no & Age groups & Frequency $(\%)$ \\
\hline 1 & $\leq 40$ & $33(27.5)$ \\
\hline 2 & $41-45$ & $39(32.5)$ \\
\hline 3 & $46-50$ & $18(15)$ \\
\hline 4 & $51-55$ & $21(17.5)$ \\
\hline 5 & $\geq 56$ & $09(7.5)$ \\
\hline
\end{tabular}

$1 B$.

\begin{tabular}{|l|l|l|}
\hline S/no & Age groups & Frequency $(\%)$ \\
\hline 1 & $\leq 50$ & $90(75)$ \\
\hline 2 & $\geq 51$ & $30(25)$ \\
\hline
\end{tabular}

Table 2. Percentage oocyte fertilization and age groupings

2.

\begin{tabular}{|l|lll|}
\hline S/NO & Age groups & $\begin{array}{l}\text { Percentage oocyte } \\
\text { fertilized }(\%)\end{array}$ & $\mathrm{X}^{2}=90.133$ \\
\cline { 1 - 1 } 1 & $\leq 50$ & 54.21 & $\mathrm{P}=0.0000$ \\
\cline { 1 - 1 } 2 & $\geq 51$ & $45.21 \%$ & \\
\hline
\end{tabular}

3.

\begin{tabular}{|l|l|l|l|}
\hline S/no & Age groups & $\begin{array}{l}\text { Percentage oocyte } \\
\text { fertilized (\%) }\end{array}$ & \multirow{2}{*}{ X $=0.405$} \\
\cline { 1 - 1 } 1 & $\leq 40$ & 54.94 & \\
\cline { 1 - 1 } 2 & $41-45$ & 49.98 & \\
\cline { 1 - 1 } 3 & $46-50$ & 62.02 & \\
\cline { 1 - 1 } 5 & $51-55$ & 46.19 & \\
\hline 5 & $\geq 56$ & 42.91 & \\
\hline
\end{tabular}

Table 4. Mean post semen wash sperm concentration among age groupings undergoing IVF cycle

\begin{tabular}{|c|c|c|c|}
\hline S/no & Age groups & $\begin{array}{l}\text { Mean semen concentration } \\
\left.\text { (x } 10^{6} / \mathrm{ml}\right)\end{array}$ & \multirow{6}{*}{$\begin{array}{l}X^{2}= \\
413.132 \\
P=0.000\end{array}$} \\
\hline 1 & \multirow{5}{*}{$\begin{array}{l}\leq 40 \\
41-45 \\
46-50 \\
51-55 \\
\geq 56\end{array}$} & \multirow{5}{*}{$\begin{array}{l}117.54( \pm 91.49) \\
113.15( \pm 73.88) \\
128.15( \pm 111.85) \\
128.50( \pm 111.58) \\
266.67( \pm 145.14)\end{array}$} & \\
\hline 2 & & & \\
\hline 3 & & & \\
\hline 4 & & & \\
\hline 5 & & & \\
\hline
\end{tabular}

Table 5. Mean post semen wash sperm motility among age groupings undergoing IVF cycle

\begin{tabular}{|l|l|l|l|}
\hline S/no & Age groups & Mean semen motility $(\%)$ & $\mathrm{X}^{2}=249.684$ \\
\cline { 1 - 1 } 1 & $\leq 40$ & $47.27( \pm 20.12)$ & $\mathrm{P}=0.000$ \\
\cline { 1 - 1 } 3 & $41-45$ & $49.46( \pm 12.61)$ & \\
\cline { 1 - 1 } 4 & $46-50$ & $41.17( \pm 18.97)$ & \\
\hline $51-55$ & $41.17( \pm 18.97)$ & \\
\hline 5 & $\geq 56$ & $37.23( \pm 18.19)$ & \\
\hline
\end{tabular}




\section{References}

[1]. American Urological Association (2008). A basic guide to male infertility: how to find out what's wrong. Retrieved June 11, 2012.

[2]. American Society for Reproductive Medicine. (2012). Optimizing natural fertility. Retrieved June 11, 2012, from http://www.asrm.org/uploadedFiles/ASRM_Content/News_and_Publications/Practice_Guidelines/committee_Opini ons/optimizing_natural_fertility(2).pdf https://hyattractions.wordpress.com/2012/11/.../male-and -femalereproductive-system...Nov 12, 2012

[3]. Andolz P, Bielsa MA, Vila J. Evolution of semen quality in North-eastern Spain: a study in 22,759 infertile men over a 36 year period. Hum Reprod. 1999; 14:731-735.

[4]. Auger J, Kunstmann JM, Czyglik F, Jouannet P. Decline in semen quality among fertile men in Paris during the past 20 years. N Engl J Med. 1995; 332:281-285.

[5]. Brahem S, Mehdi M, Elghezal H, Saad H. The effects of male aging on semen quality, sperm DNA fragmentation and chromosomal abnormalities in an infertile population. J Assist Reprod Genet. 2011 May; 28(5): 425-432.

[6]. Harris I.D, Fronczak C, Roth L, Meacham R.B. Fertility and the Aging Male. Rev Urol. 2011; 13(4): e184e190.

[7]. Jeulin C, Serres C, Jouannet P. The effects of centrifugation, various synthetic media and temperature on the motility and vitality of human spermatozoa. Reprod Nutr Dev 1982; 22:81.

[8]. Jose-Miller, A. B., Boyden J. W., \& Frey, K. A. (2007). Infertility. American Family Physician, 75, 849-856.

[9]. Male Infertility Best Practice Policy Committee of the American Urological Association \& Practice Committee of the American Society of Reproductive Medicine. (2006).Report on optimal evaluation of the infertile male. Fertility and Sterility, 86, S202-S209.

[10]. Michelmann H.W. Minimal criteria of sperm quality for insemination and IVF therapy. Int J Androl. 1995 Dec; 18 Suppl 2:81-7.

[11]. Morshedi M. New 2010 WHO Standards (5th Edition) for the Evaluation of Human Semen. WHO semen analysis review. https://www.aab.org/images/aab/pdf/2014/CRBPPT14/WHO.pdf Ok E. K, Doğan O.E, Okyay R.E, Gülekli B. The effect of post-wash total progressive motile sperm count and semen volume on pregnancy outcomes in intrauterine insemination cycles: a retrospective study. J Turk Ger Gynecol Assoc. 2013; 14(3): 142-145.

[12]. Practice Committee of the American Society for Reproductive Medicine in collaboration with the Society for Reproductive Endocrinology and Infertility. (2008). Optimizing natural fertility. Fertility and Sterility, 90(5, suppl), S1-S6.

https://en.wikipedia.org/wiki/Male_reproductive_system (https://prezi.com/i0z5miveenwg/human-malereproductive-system/ Oct 1, 2013).

[13]. Schwartz D, Mayaux MJ, Spira A, et al. Semen characteristics as a function of age in 833 fertile men. Fertil Steril. 1983; 39:530-535.

[14]. Sloter E, Schmid TE, Marchetti F, et al. Quantitative effects of male age on sperm motion. Hum Reprod. 2006; 21:2868-2875.

[15]. Wu Y, Kang X, Zheng H, Liu H, Liu J. Effect of Paternal Age on Reproductive Outcomes of In Vitro Fertilization. PLoS One. 2015; 10(9): e0135734.

www.post-nigeria.com/common-causes-of-infertility-in-men-by-damilola-faleke/May 20, 2015. 